\title{
Brigatinib for anaplastic lymphoma kinase-tyrosine kinase inhibitor naïve anaplastic lymphoma kinase-positive advanced non-small cell lung cancer: an effective but still broken option
}

\author{
Biagio Ricciuti ${ }^{1}$, Giuseppe Lamberti ${ }^{2}$, Fausto Roila ${ }^{1}$, Giulio Metro ${ }^{1}$ \\ ${ }^{1}$ Medical Oncology, Santa Maria della Misericordia Hospital, Azienda Ospedaliera di Perugia, Perugia, Italy; ${ }^{2}$ Department of Experimental, \\ Diagnostic and Specialty Medicine, Policlinico di Sant'Orsola University Hospital, Bologna, Italy \\ Correspondence to: Giulio Metro, MD. Medical Oncology, Santa Maria della Misericordia Hospital, Azienda Ospedaliera di Perugia, via Dottori, 1, \\ 06156, Perugia, Italy. Email: giulio.metro@yahoo.com. \\ Provenance: This is an invited article commissioned by the Section Editor Hengrui Liang (Department of Thoracic Surgery, Guangzhou Medical \\ University, Guangzhou, China). \\ Comment on: Camidge DR, Kim HR, Ahn MJ, et al. Brigatinib versus Crizotinib in ALK-Positive Non-Small-Cell Lung Cancer. N Engl J Med \\ 2018;379:2027-39.
}

Submitted Apr 04, 2019. Accepted for publication Apr 28, 2019.

doi: $10.21037 /$ tlcr.2019.04.15

View this article at: http://dx.doi.org/10.21037/tlcr.2019.04.15

Rearrangements of the anaplastic lymphoma kinase (ALK) gene occur in approximately $5 \%$ of non-small cell lung cancers (NSCLCs), in which they identify a distinct subtype of lung tumor that shows exquisite sensitivity to therapy with ALK tyrosine kinase-inhibitors (ALK-TKIs) (1).

Despite durable responses to the first-generation ALKTKI crizotinib, the development of acquired resistance occurs in virtually all patients (2). From a biological standpoint, resistance to crizotinib develops either by 'on target' (ALK secondary mutations in the tyrosine kinase domain or ALK amplification) or 'off target' mechanisms (activation of signaling pathways other than ALK) (3). The novel second-generation ALK-TKI brigatinib has shown preclinical activity against a wide spectrum of ALK secondary mutations associated with resistance to crizotinib, and consistently, it proved to be clinically effective in crizotinib-refractory patients (4-6). Against this background, in September 2018, Camidge and colleagues reported in the New England Fournal of Medicine the eagerly awaited results of the 'ALTA-1L' trial, which compared brigatinib with crizotinib in ALK-TKI naïve patients with ALK-positive advanced NSCLC (7).

\section{Study overview}

'ALTA-1L' is a multicenter, randomized, open-label, phase 3 trial that allocated 275 patients with ALK-TKI naïve ALK-positive advanced NSCLC in a 1:1 ratio to either brigatinib $180 \mathrm{mg}$ once daily (after a 7 days lead-in phase at $90 \mathrm{mg} ; \mathrm{N}=137$ ) or crizotinib $250 \mathrm{mg}$ twice daily $(\mathrm{N}=138)$ (7). Crossover to brigatinib was allowed for patients in the crizotinib arm, upon confirmation of disease progression by blinded independent review assessment. The primary endpoint of the study was progression-free survival (PFS), while secondary endpoints included objective response rate (ORR), intracranial ORR (IORR), intracranial PFS, and overall survival. The first pre-specified interim analysis was performed at $50 \%$ of expected events (99/198). With a median follow-up of 11.0 months in the brigatinib group and 9.3 months in the crizotinib group, blinded independent review-assessed PFS was significantly longer for brigatinib [median PFS not reached versus 9.8 months (95\% CI, 9.0-12.9 months), respectively], with an estimated 12 -month progression-free rate of $67 \%$ (95\% CI, $56-$ $75 \%$ ) versus $43 \%$ (95\%, CI, 32-53\%), respectively, and a hazard ratio (HR) for progression or death of 0.49 in favor of brigatinib [(95\% CI, 0.33-0.74), $\mathrm{P}<0.001]$. The subgroup analysis showed that brigatinib was superior to crizotinib across several clinical characteristics, including performance status ( 0 or 1$)$, presence of brain metastases (BMs) at baseline, and prior exposure to chemotherapy for advanced disease (the study allowed 
patients pretreated with no more than one prior systemic anticancer therapy). Of note, the HR for progression or death in favor of brigatinib was 0.35 (95\% CI, 0.14-0.85) and 0.55 (95\% CI, $0.34-0.88)$ in patients who had or had not received prior chemotherapy, respectively. With regard to secondary endpoints, brigatinib yielded a higher ORR by blinded independent review in the intentionto-treat population, being $71 \%$ (95\% CI, $62-78 \%$ ) for brigatinib and $60 \%$ (95\% CI, 51-68\%) for crizotinib. In addition, response to treatment was more durable in the brigatinib arm than in the crizotinib arm [median duration of response not reached versus 11.1 months (95\% CI, 9.2-not reached months), respectively]. However, the most striking difference between the two treatment arms was observed in the subgroup of patients with BMs at baseline. Overall, 90 patients out of 275 had BMs (32.7\%) by blinded independent review, of whom 18/43 (41.9\%) and 21/47 (44.7\%) had measurable BMs according to RECIST $\mathrm{v} 1.1$ in the brigatinib and crizotinib groups, respectively. Of note, in this subgroup the confirmed IORR was 78\% (95\% CI, 52-94\%) for brigatinib and 29\% (95\% CI, 11-52\%) for crizotinib. A similarly higher difference in activity against central nervous system (CNS) disease was observed in the overall population with BMs, with a confirmed IORR of $67 \%$ (95\% CI, 51-81\%) in the brigatinib arm as compared to $17 \%$ (95\% CI, $8-31 \%$ ) in the crizotinib arm. Interestingly, when the authors carried out an exploratory competing-risks analysis of intracranial or systemic disease progression and death, they found that the cause-specific HR for time to progression of intracranial disease was 0.30 (95\% CI, 0.15-0.60) in favor of brigatinib. Overall survival data were not mature at the time of the analysis, 1-year rate of survival being $85 \%$ (95\% CI, 76-91\%) for brigatinib and $86 \%$ (95\% CI, 77-91\%) for crizotinib.

No unexpected toxicities occurred during the trial, and no treatment-related deaths were reported. However, some significant differences in toxicities were noted between the two study arms: regardless of the severity, increased blood creatine kinase level, cough, hypertension and increased lipase/amylase levels were more common in the brigatinib arm, while gastrointestinal symptoms (nausea, vomiting, diarrhea, constipation), peripheral edema, increased alanine aminotransferase level, decreased appetite, photopsia, dysgeusia, and visual impairment were more common in patients receiving crizotinib. Grade 3 or higher interstitial lung disease or pneumonitis occurred in $3 \%$ of patients randomized to brigatinib and the rate of any grade interstitial lung disease or pneumonitis was $3 \%$ in patients who crossed over from crizotinib to brigatinib.

\section{Place in therapy}

Although 'ALTA-1L' has clearly shown that brigatinib improves PFS compared to crizotinib in ALK-TKI naïve ALK-positive NSCLC patients, the extent of this benefit remains to be determined as the follow-up is still immature. Brigatinib has previously demonstrated to be effective at progression on crizotinib, with some signs of antitumor activity also when administered after the second-generation ALK-inhibitor alectinib $(5,6,8)$. In the latest update of the 'ALTA' phase 2 trial of crizotinib-refractory patients, brigatinib has shown an ORR of $56 \%$ with a remarkable median PFS of 16.7 months, which emerges as the longest PFS of any ALK inhibitor to be reported for patients who have progressed on crizotinib (6). However, whether this highest-ranking position in the post-crizotinib setting will translate into a first-ranking benefit in ALK-TKI naïve patients is yet to be determined. Preclinical data have shown that brigatinib has the broadest in vitro coverage against secondary ALK resistance mutations compared to the other clinically available second-generation ALK inhibitors, namely alectinib and ceritinib $(9,10)$. If expectations will be met, it can be argued that moving brigatinib in the first-line setting may significantly delay the emergence of resistance to treatment. However, the recent phase 3 'ALEX' trial has established alectinib as the new preferred first-line option for patients with ALK-positive advanced NSCLC $(11,12)$. In 'ALEX', alectinib excelled over crizotinib in term of median PFS [34.8 versus 10.9 months, respectively; HR $=0.43$ (95\% CI, 0.32-0.58)], and also provided evidence for a greater intracranial activity [median PFS in patients with BMs was 27.7 versus 7.4 months, respectively; HR $=0.35$ (95\% CI, 0.22-0.56)]. Therefore, data from 'ALTA-1L' add to the current standard of care as they expand to brigatinib the treatment options for ALK-TKI naïve ALK-positive NSCLCs. Despite a shorter followup, the early 'ALTA-1L' results for brigatinib as compared with crizotinib appear similar to clinical outcomes from the 'ALEX' trial (Table 1). However, at present time there are no data favoring the use of brigatinib over alectinib as firstline treatment or vice versa. As alectinib has already moved into the up-front setting, the most important question that we need to address is what are the most active treatments for patients who progress on alectinib. In a recent retrospective study of 22 alectinib-refractory ALK-positive patients treated with brigatinib, Lin $e t a l$. reported an ORR 
Table 1 Comparison between the ALEX and the ALTA-1L phase III clinical trials

\begin{tabular}{|c|c|c|c|c|}
\hline Drug & \multicolumn{2}{|c|}{ ALEX } & \multicolumn{2}{|c|}{ ALTA-1L } \\
\hline Number of patients & 151 & 152 & 138 & 137 \\
\hline Median PFS & 10.9 & 34.8 & 9.8 & NR \\
\hline PFS HR (95\% Cl) & \multicolumn{2}{|c|}{$0.43(0.38-0.52)$} & \multicolumn{2}{|c|}{$0.49(0.33-0.74)$} \\
\hline PFS in patients with BMs & 7.4 & NR & 5.6 & NR \\
\hline PFS HR $(95 \% \mathrm{Cl})$ with BMs & \multicolumn{2}{|c|}{$0.40(0.25-0.64)$} & \multicolumn{2}{|c|}{$0.20(0.09-0.46)$} \\
\hline PFS in patients without BMs & 14.8 & NR & 11.1 & NR \\
\hline PFS HR (95\% Cl) without BMs & \multicolumn{2}{|c|}{$0.51(0.33-0.80)$} & \multicolumn{2}{|c|}{$0.72(0.44-1.18)$} \\
\hline
\end{tabular}

PFS, progression-free survival; HR, hazard ratio; ORR, objective response rate; NR, not reached; BMs, brain metastases.

of $16.7 \%$ (3/18 patients with baseline measurable disease) and a median PFS of 4.4 months (8). This study showed that clinical outcomes on brigatinib following alectinib are considerably lower as compared to the crizotinibrefractory setting, which is not surprising given the similar ALK inhibitory potency and activity against CNS disease of alectinib and brigatinib. Likewise, the 'ASCEND-9' trial evaluated the clinical activity of another clinically available second-generation ALK-TKI such as ceritinib in patients who had progressed on alectinib. Among 20 alectinibpretreated patients enrolled in this study, the ORR with ceritinib was $25 \%$, and median PFS was dismal, being only 3.7 months (13). However, these data also indicate that, according to the mechanism of resistance, there might be room for brigatinib or ceritinib in a very selected subset of alectinib-refractory patients. We know that the presence of specific ALK resistance mutations affect the clinical sensitivity to other second-generation ALK-TKIs in alectinib-refractory tumors (8). Importantly, secondary ALK resistance mutations are most frequently detected after second-generation ALK-TKIs rather than after crizotinib ( $50 \%$ versus $\sim 20 \%$ ), and each second-generation ALKTKI appears to generate a distinct spectrum of resistance mutations (10). After alectinib, the most common resistance mutations include G1202R (30\%) followed by I1171N, and V1180L. Although preclinical studies indicate that brigatinib maintains a good activity against the $I 1171 \mathrm{~N}$ and V1180L mutations, data regarding the activity of brigatinib against the G1202R mutation are still controversial $(9,10)$. Although anecdotical responses to brigatinib have been reported in patients with the G1202R mutation, in vitro data suggest that the G1202R mutations remains the relatively most resistant mutation to brigatinib $\left(\mathrm{IC}_{50}=184 \mathrm{nmol} / \mathrm{L}\right.$ ) $(5,9)$. On the other hand, the third-generation ALK-TKI lorlatinib has shown activity against all of the known ALK resistance mutations, including G1202R, and now it is the preferred option in the setting of resistance to alectinib, as discussed below (14).

\section{Future directions}

In 'ALTA-1L', brigatinib demonstrated superior efficacy compared with crizotinib in terms of both systemic and intracranial disease. This trial establishes brigatinib as a novel first-line option for ALK-TKI naïve ALK-positive NSCLC, along with alectinib. Having said that, the landscape of treatment of ALK-positive NSCLC is still evolving and several novel ALK-TKIs are under preclinical and clinical development, including, but not limited to, ensartinib, entrectinib and lorlatinib (15). Particularly, lorlatinib is a highly potent, selective ALK inhibitor that is able to surpass the blood-brain barrier and to be operate against most known ALK resistance mutations developing after exposure to crizotinib and second-generation ALK inhibitors, including G1202R (14). Consistently, in a phase 2 trial lorlatinib has recently demonstrated a meaningful overall and intracranial activity both in ALK-TKI naïve patients with ALK-positive NSCLC, as well as in those who had progressed on crizotinib and second-generation ALK inhibitors (16). Importantly, antitumor activity was seen across a range of ALK resistance mutations, including G1202R and G1202del, and in the group of patients pretreated with $\geq 1$ ALK-TKIs the greatest 

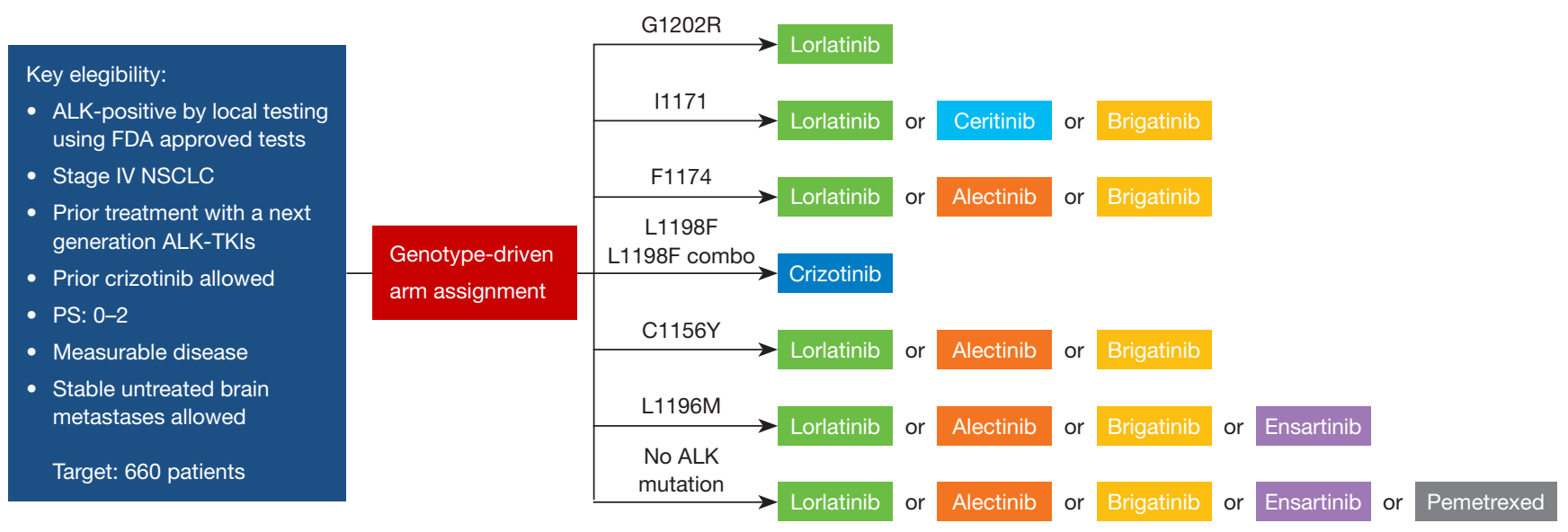

Figure 1 Flow chart of the ALK Master Protocol. ALK, anaplastic lymphoma kinase; FDA, Food and Drug Administration; NSCLC, nonsmall-cell lung cancers; TKI, tyrosine kinase-inhibitor; PS, performance status.

benefit was observed in patients who had been documented with ALK resistance mutations by either plasma or tissue genotyping (17). More importantly, and not surprisingly, the magnitude of the clinical benefit from lorlatinib was greater in ALK-TKI naïve patients. These data indicate that lorlatinib might provide an effective therapeutic first-line option, which is the rationale of the currently ongoing phase 3 'CROWN' trial (NCT03052608) comparing lorlatinib versus crizotinib in treatment naïve ALK-positive NSCLCs.

As more treatment options are becoming available for these patients and the optimal sequence is still unclear, the US National Cancer Institute (NCI) has sponsored a biomarker-driven study which will help us to understand how to optimize patient selection for ALK-TKIs. The NCINRG ALK Master Protocol (NCT03737994) is a phase 2 trial designed for patients with advanced ALK-positive NSCLC, in which the different mutations will direct the given treatment and sequence (Figure 1). Hopefully, this study will lead us to a more comprehensive understanding of the proper management of these patients.

\section{Acknowledgments}

None.

\section{Footnote}

Conflicts of Interest: The authors have no conflicts of interest to declare.

\section{References}

1. Metro G, Bellezza G, Puma F, et al. How might treatment of ALK-positive non-small cell lung cancer change in the near future? Expert Rev Anticancer Ther 2016;16:997-9.

2. Metro G, Tazza M, Matocci R, et al. Optimal management of ALK-positive NSCLC progressing on crizotinib. Lung Cancer 2017;106:58-66.

3. Lin JJ, Riely GJ, Shaw AT. Targeting ALK: precision medicine takes on drug resistance. Cancer Discov 2017;7:137-55.

4. Ali R, Arshad J, Palacio S, et al. Brigatinib for ALKpositive metastatic non-small-cell lung cancer: design, development and place in therapy. Drug Des Devel Ther 2019;13:569-80.

5. Kim DW, Tiseo M, Ahn MJ, et al. Brigatinib in patients with crizotinib-refractory anaplastic lymphoma kinasepositive non-small-cell lung cancer: a randomized, multicenter phase II trial. J Clin Oncol 2017;35:2490-8.

6. Camidge DR, Tiseo M, Ahn MJ, et al. P3.02a-013 Brigatinib in Crizotinib-Refractory ALK+ NSCLC: Central Assessment and Updates from ALTA, a Pivotal Randomized Phase 2 Trial. J Thorac Oncol 2017;12:S1167-9.

7. Camidge DR, Kim HR, Ahn MJ, et al. Brigatinib versus Crizotinib in ALK-Positive Non-Small-Cell Lung Cancer. N Engl J Med 2018;379:2027-39.

8. Lin JJ, Zhu VW, Schoenfeld AJ, et al. Brigatinib in patients with alectinib-refractory ALK-positive NSCLC. J 
Thorac Oncol 2018;13:1530-8.

9. Zhang S, Anjum R, Squillace R, et al. The potent ALK inhibitor brigatinib (AP26113) overcomes mechanisms of resistance to first- and second-generation ALK inhibitors in preclinical models. Clin Cancer Res 2016;22:5527-38.

10. Gainor JF, Dardaei L, Yoda S, et al. Molecular mechanisms of resistance to first- and second-generation ALK inhibitors in ALK-rearranged lung cancer. Cancer Discov 2016;6:1118-33.

11. Peters S, Camidge DR, Shaw AT, et al. Alectinib versus crizotinib in untreated ALK-positive non-small-cell lung cancer. N Engl J Med 2017;377:829-38.

12. Camidge DR, Peters S, Mok T, et al. Updated efficacy and safety data from the global phase III ALEX study of alectinib versus crizotinib in untreated advanced ALK+ NSCLC. J Clin Oncol 2018 36:abstr 9043.

13. Hida T, Seto T, Horinouchi H, et al. Phase II study of ceritinib in alectinib-pretreated patients with anaplastic lymphoma kinase-rearranged metastatic non-smallcell lung cancer in Japan: ASCEND-9. Cancer Sci 2018;109:2863-72.

Cite this article as: Ricciuti B, Lamberti G, Roila F, Metro G. Brigatinib for anaplastic lymphoma kinase-tyrosine kinase inhibitor naïve anaplastic lymphoma kinase-positive advanced non-small cell lung cancer: an effective but still broken option. Transl Lung Cancer Res 2019;8(Suppl 4):S378-S382. doi: 10.21037/tlcr.2019.04.15
14. Johnson TW, Richardson PF, Bailey S, et al. Discovery of (10R)-7-amino-12-fluoro-2,10,16-trimethyl-15-oxo10,15,16,17-tetrahydro-2H-8,4-(metheno)pyrazolo[4,3-h] [2,5,11]-benzoxadiazacyclotetradecine-3-carbonitrile (PF-06463922), a macrocyclic inhibitor of anaplastic lymphoma kinase (ALK) and c-ros oncogene 1 (ROS1) with preclinical brain exposure and broad-spectrum potency against ALK-resistant mutations. J Med Chem 2014;57:4720-44.

15. Ricciuti B, De Giglio A, Mecca C, et al. Precision medicine against ALK-positive non-small cell lung cancer: beyond crizotinib. Med Oncol 2018;35:72.

16. Solomon BJ, Besse B, Bauer TM, et al. Lorlatinib in patients with ALK-positive non-small-cell lung cancer: results from a global phase 2 study. Lancet Oncol 2018;19:1654-67.

17. Shaw AT, Solomon BJ, Besse B, et al. ALK Resistance Mutations and Efficacy of Lorlatinib in Advanced Anaplastic Lymphoma Kinase-Positive Non-Small-Cell Lung Cancer. J Clin Oncol 2019;37:1370-9. 